\title{
System offers rapid, low-cost measurement of thickness and optical constants of thin films
}

\section{The pitch}

Thin-film solar cells, liquid-crystal displays, light-emitting diodes, and many other thin-film-containing products require inline metrology for the rapid, reliable, and low-cost control of the thickness and optical constants (e.g., the refractive index and extinction coefficient) of the thin films. This requisite is particularly crucial in thin-film solar cell production where the absorber layerfor example, amorphous silicon (aSi) or $\mathrm{CuInGaSe}_{2}$ (CIGS) - must be precisely controlled: A deviation in the absorber composition directly affects the efficiency of the solar cells. Thickness control also requires striking a balance: If the film is too thick, the manufacturing time and cost of materials increase; if it is too thin, light is not fully absorbed and the cell efficiency drops.

Because of the size of solar panels, the uniformity of the layers is a concern. One approach is to use a large number of measurement probes to simultaneously take measurements at different points on the panel. Spectroscopic ellipsometers (SEs) are frequently used by researchers for this application. But their low measurement speed and high cost make this instrument unsuitable for production inline applications. Alternatively, spectroscopic reflectometry, which offers rapid and relatively lower cost measurement, has been traditionally limited only to thickness measurement. However, Semiconsoft's fiberoptics MProbe thin-film measurement system has been optimized to achieve exceptional precision and analysis capabilities.

\section{The technology}

Spectroscopic reflectance is a powerful nondestructive method for thin-film measurement. With proper optimization, the use of fiberoptics-based systems can significantly reduce cost without sacrificing measurement performance. The combination of high measurement precision and appropriate data analysis makes a

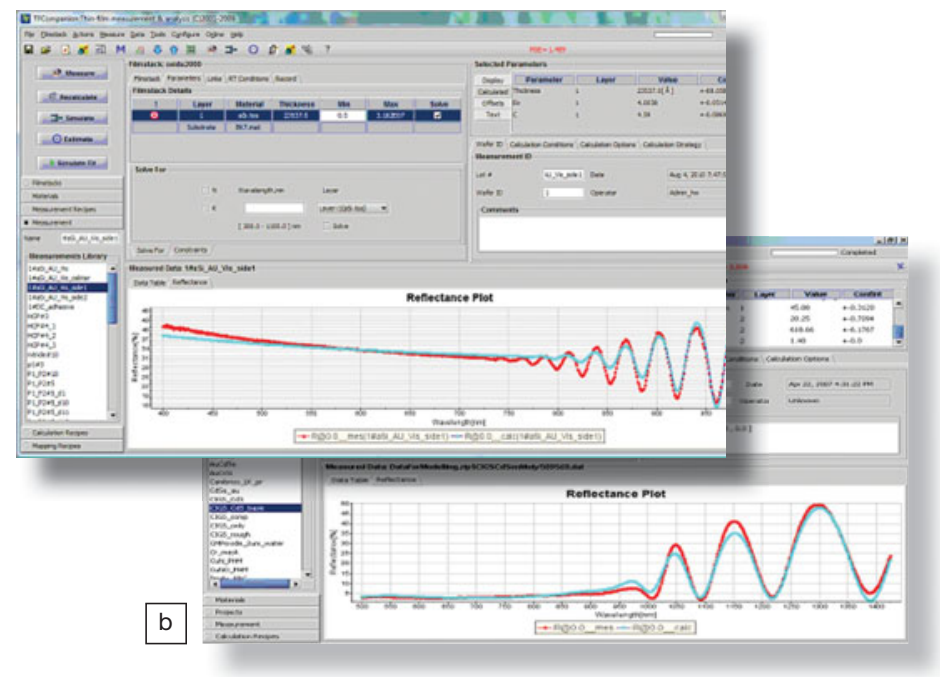

Figure 1(a) Measurement of aSi (fit of model to measured data). The thickness, $2.55 \mu \mathrm{m}$, and the optical constants spectra were determined using the Tauc-Lorentz approximation for the aSi dispersion. (Measured parameters $E_{0}$ and $C$ are in the table). (b) Measurement of CdS/CIGS/Mo filmstack (fit of model to measured data).

Measured parameters: CIGS thickness $(1.54 \mu \mathrm{m})$, CdS thickness $(77 \mathrm{~nm})$, surface roughness $(61 \mathrm{~nm})$, CIGS composition: $45 \%(\mathrm{Ga} /(\mathrm{ln}+\mathrm{Ga}))$, void fraction in CdS roughness layer: $20 \%$. possible the measurement of the thicknesses and optical constants of a wide range of filmstacks.

There are several common misconceptions about the capabilities of spectroscopic reflectance as applied to thinfilm measurement. It is assumed, for example, that by using this technique only thickness can be determined (the optical constants: refractive index, $n$, and extinction coefficient, $k$, need to be known), that it has lower precision compared with ellipsometry, and that only one or two thicknesses can be determined simultaneously. These misconceptions reflect the traditional/historical use of this technique and, indeed, the capabilities of the majority of commercially available instruments. However, they are not limitations of the technique but the way it is used and how the data are interpreted (i.e., the software analysis).

Both techniques can measure complex multilayer filmstacks and can determine the thickness and optical constants ( $n, k$ values) of the materials.

The key to the successful measurement of materials like aSi and CIGS with spectroscopic reflectometry is the use of proper parameterization of the $n, k$ spectra. In the case of aSi, the Tauc-Lorentz approximation (refers to the optical properties of amorphous materials) and in the case of CIGS, compound material approximation. An example of the results of aSi measurement is shown in Figure 1a and an example of the results of $\mathrm{CdS} / \mathrm{CIGS} / \mathrm{Mo}$ measurement is shown in Figure 1b. Using spectroscopic reflectometry, complete thin-film solar filmstacks and many other applications can be measured rapidly and at low cost.

\footnotetext{
Opportunities

Semiconsoft, Inc. is currently pursuing opportunities for the integration of its sensors for inline metrology in research and development (R\&D) and production lines for thin-film solar materials, architectural glass, and similar products.

Source: Leo Asinovski,

Semiconsoft, Inc., 83 Pine Hill Road, Southborough, MA 01772 USA; tel. 617-388-6832; fax 508-858-5473; e-mail Leo.Asinovski@semiconsoft.com; and www.semiconsoft.com.
} 